\title{
Outcomes and care practices for preterm infants born at less than 33 weeks' gestation: a quality-improvement study
}

\author{
Shoo K. Lee MBBS PhD, Marc Beltempo MD, Douglas D. McMillan MD, Mary Seshia MBChB, Nalini Singhal MD, \\ Kimberly Dow MD, Khalid Aziz MBBS MEd, Bruno Piedboeuf MD, Prakesh S. Shah MD; on behalf of the \\ Evidence-based Practice for Improving Quality Investigators*
}

Cite as: CMAJ 2020 January 27;192:E81-91. doi: 10.1503/cmaj.190940

${ }^{*}$ The full list of Evidence-based Practice for Improving Quality Investigators can be found at the end of the article.

See related article at www.cmaj.ca/lookup/doi/10.1503/cmaj.200008

\begin{abstract}
BACKGROUND: Preterm birth is the leading cause of morbidity and mortality in children younger than 5 years. We report the changes in neonatal outcomes and care practices among very preterm infants in Canada over 14 years within a national, collaborative, continuous quality-improvement program.
\end{abstract}

METHODS: We retrospectively studied infants born at 23-32 weeks' gestation who were admitted to tertiary neonatal intensive care units that participated in the Evidence-based Practice for Improving Quality program in the Canadian Neonatal Network from 2004 to 2017. The primary outcome was survival with- out major morbidity during the initial hospital admission. We quantified changes using process-control charts in 6-month intervals to identify specialcause variations, adjusted regression models for yearly changes, and interrupted time series analyses.

RESULTS: The final study population included 50831 infants. As a result of practice changes, survival without major morbidity increased significantly (56.6\% [669/1183] to $70.9 \%$ [1424/2009]; adjusted odds ratio [OR] 1.08, 95\% confidence interval $[\mathrm{Cl}] 1.06-1.10$, per year) across all gestational ages. Survival of infants born at 23-25 weeks' gestation increased $(70.8 \%$ [97/137] to $74.5 \%$ [219/294]; adjusted OR 1.03, 95\% Cl 1.02-1.05, per year). Changes in care practices included increased use of antenatal steroids (83.6\% [904/1081] to $88.1 \%$ [1747/1983]), increased rates of normothermia at admission (44.8\% [520/1160] to $67.5 \%[1316 / 1951])$ and reduced use of pulmonary surfactant (52.8\% [625/1183] to 42.7\% [857/2009]).

INTERPRETATION: Network-wide qualityimprovement activities that include better implementation of optimal care practices can yield sustained improvement in survival without morbidity in very preterm infants.

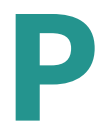

reterm birth is rising rapidly around the world (incidence ranges from $5 \%$ to $18 \%$ ) and is the leading cause of cerebral palsy, autism, developmental delay and death among children younger than 5 years. ${ }^{1,2}$ Among preterm infants, those born before 33 weeks' gestation are at highest risk of mortality and morbidity, with resultant financial and social costs for families and society. ${ }^{3,4}$ The Canadian Neonatal Network was established in 1995 to benchmark and improve national neonatal outcomes in the setting of a health system in which hospital care and physicians are publicly funded.

The Canadian Neonatal Network includes all tertiary level neonatal intensive care units (NICUs) across Canada, whose population of 36 million with 360000 annual births is served by regionalized perinatal care systems of maternity units and 31 tertiary level NICUs. In 2003, we launched a national Evidencebased Practice for Improving Quality (EPIQ) program within the Canadian Neonatal Network. This program is unique because it is national in scale, shows how self-learning systems can work and has been sustained over time.

In this study, we report the impact of the EPIQ program on outcomes of very preterm infants born at 23-32 weeks' gestation in Canada from 2004 to 2017, in line with the Standards for Quality Improvement Reporting Excellence 2.0 guidelines. $^{5}$ 


\section{Methods}

The EPIQ program is a collaborative, multifaceted qualityimprovement approach that combines iterative learning techniques using Plan-Do-Study-Act cycles of rapid change with a process to facilitate quality improvement through benchmarking, best-practice consensus, engagement of front-line staff and mutual learning via networking. In addition, we used institutionspecific data to target practices with high attributable risk of adverse outcomes for interventions and to minimize use of inefficient "shotgun" approaches for quality improvement. The program emphasizes changing individual behaviour and organizational culture. ${ }^{6}$ We established a central resource for knowledge and data management, assessed organizational culture and behaviour, created multidisciplinary teams that included parents and staff, and used a train-the-trainer approach to engage staff and implement Plan-Do-Study-Act cycles at participating NICUs.

\section{Study population}

We conducted a retrospective cohort study involving infants born from $23+0$ to $32+6$ weeks' gestation and admitted to participating NICUs between Jan. 1, 2004, and Dec. 31, 2017. Canada's NICUs range in size from 20 to 83 (median 30) beds, and care for 102 to 1200 admissions per year and 0 to 7000 births annually. ${ }^{7}$ Twenty-two NICUs collected data from 2004 and another 7 commenced in 2008.

Over the 14-year study period, there were 3 main epochs of the EPIQ program. All units that were part of the Canadian Neonatal Network were offered the opportunity to participate in the program. Twelve (out of 17) tertiary level NICUs voluntarily participated in Epoch 1 (2004-2008), and 25 (out of 29) participated in Epochs 2 (2009-2012) and 3 (2013-2017). We included all eligible infants admitted to each participating site. We excluded infants who were moribund on admission, had major congenital anomalies, were missing gestational age or birth weight, or were admitted more than 7 days after birth. Our cohort included more than $80 \%$ of eligible infants born in Canada as some low-risk infants were cared for in non-ICUs that did not participate in the EPIQ program.

\section{Data collection}

The Canadian Neonatal Network provides standardized training for all dedicated data abstractors in the respective institutions. A standard data dictionary and data-collection protocol is followed by all abstractors. ${ }^{8}$ Anonymized patient data are entered electronically into a purpose-built program, with error checks at entry. The Canadian Neonatal Network database has shown high reliability and internal consistency. ${ }^{9}$ The first (S.K.L.) and last (P.S.S.) authors had full access to all data and analyses. A statistician (X.Y.Y.) at the Maternal-Infant Care Research Centre, Sinai Health System, conducted the data analysis.

\section{Variable definitions}

We calculated gestational age hierarchically from best estimate based on the date of in vitro fertilization, early prenatal ultrasonography, last menstrual period, obstetric estimate and pediatric estimate. We used Canadian standards to classify infants as small for gestational age (<10th percentile). ${ }^{10} \mathrm{We}$ collected data on specific care practices, including delivery in a tertiary care unit (inborn), use of antenatal steroids (partial or complete), normothermia on admission (between $36.5^{\circ} \mathrm{C}$ and $37.5^{\circ} \mathrm{C}$ ), use of pulmonary surfactant, cesarean birth and use of systemic postnatal steroids.

\section{Key practice changes}

Our group has previously described specific changes in practice, process measures and results of each epoch. ${ }^{11-14}$ Key timelines and the 5 most common practice changes are reported in Figure 1. In Epoch 1, they included interventions targeted to reduce bronchopulmonary dysplasia and nosocomial infection, whereas in Epochs 2 and 3, the interventions targeted all major morbidity outcomes described below.

\section{Outcomes}

For this analysis, the primary outcome was survival to NICU discharge without major morbidities, selected based on frequency and potential for long-term neurodevelopmental impact. Major morbidity was defined as 1 or more of late-onset sepsis, necrotizing enterocolitis, bronchopulmonary dysplasia, severe retinopathy of prematurity, or severe neurologic injury. We defined bronchopulmonary dysplasia as need for supplemental oxygen at 36 weeks' postmenstrual age, or time of discharge or transfer. ${ }^{15}$ We defined severe neurologic injury as grade III or IV intraventricular hemorrhage according to Papile and colleagues ${ }^{16}$ or periventricular echogenicity. We defined retinopathy of prematurity as stage III or greater in at least 1 eye according to the international classification, ${ }^{17}$ or need for treatment. We defined necrotizing enterocolitis as stage 2 or greater diagnosed according to Bell's criteria. ${ }^{18}$ We defined late-onset sepsis as positive blood or cerebrospinal fluid culture in a symptomatic neonate after 2 days of age. Secondary outcomes included individual components of the primary outcome and early-onset sepsis, defined as positive blood or cerebrospinal fluid culture in a symptomatic neonate in the first 2 days after birth.

\section{Statistical analysis}

We used 3 statistical methods: process-control charts evaluating common and special-cause variations, trend analyses and interrupted time series analyses. We used process-control methods to describe, detect and understand changes in processes of care and outcomes. We plotted crude rates using 6-month periods to provide sufficient time points for processcontrol chart analysis while maintaining more than 100 observations for subgroups. ${ }^{19}$ For each graph, upper and lower control limits were determined using standard statistical process-control parameters ( \pm 3 standard deviations) using the 14-year data. We had not preplanned to revise or "lock" the limits of the process-control charts because no data were available to establish baseline rates, and we aimed to have 24 data points. We identified special-cause variation in outcomes (significant changes that are not part of random variation) as any 


\section{Nosocomial infection}

- Strategic placement of cleanser dispensers in NICUs

- Use of $2 \%$ aqueous chlorhexidine for skin antisepsis

- Restrict number of skin breaks per patient

- For rule out of sepsis, discontinue antibiotics if 36-48-hour cultures are negative

\section{Bronchopulmonary dysplasia}

- Prophylactic surfactant for infants born $<28$ weeks

- Restrict hand ventilation

- Adopt normocarbia policy (keep $\mathrm{PCO}_{2}$ between 40 and $55 \mathrm{~mm} \mathrm{Hg}$ )

- Target oxygen saturation between $88 \%$ and $92 \%$

- Early parenteral protein and lipid nutrition $<4$ hours after birth
- QI training and dissemination of practice change guidelines to non-EPIQ NICUs
- Evaluating the improvements in NICUs that did not participate in EPIQ-1 trial

\section{Nosocomial infection}

- Central line management, bundle

- Hand hygiene

- Central line management, single intervention

- Education, audit, teambuilding

- Reduce skin breaks

- Skin-to-skin care

- Checklist for central lines

\author{
Bronchopulmonary dysplasia \\ - Use of T-piece resuscitator \\ - Enhancing or ensuring early \\ use of continuous positive \\ airway pressure \\ - Controlled oxygen use or \\ review of oxygen saturation \\ - Early use of surfactant \\ and/or early extubation \\ - Ventilator weaning algorithm \\ - Prophylactic surfactant
}

\section{Severe neurologic injury}

- Delayed cord clamping

- Use of antenatal magnesium sulphate

- Minimal use of volume expanders

- Minimum use of inotropes

\section{Retinopathy of prematurity}

- Controlled oxygen use

- Improved screening protocol
Necrotizing enterocolitis

- Feeding guidelines

- Early feeding

- Use of donor milk

- Colostrum or enhanced expressed breast milk

- Early total parental nutrition

- Hold enteral feeds during erythrocyte transfusion

- Collection of outcome data at 18 months corrected age and 36 months chronological age

- Continuous quality improvement; review of bundles to focus efforts; implement practice updates
- Implementation of sentinel event reviews

- Detailed site visits
- Driving force to reach an incidence of near zero in 5 major morbidities over a 3-year intervention period

Figure 1: Timeline of key practice changes (during Epoch 1 [2004-2008], Epoch 2 [2009-2012] and Epoch 3 [2013-2017]) implemented by participating hospitals using the EPIQ method. The 5 most commonly implemented interventions were central venous catheter management bundles in different forms, development and implementation of guidelines for respiratory management of a neonate who is receiving invasive or noninvasive respiratory support, development and implementation of feeding guidelines, controlled use of oxygen, and neurologic injury prevention bundle. Note: EPIQ = Evidence-based Practice for Improving Quality, NICU = neonatal intensive care unit, PHSI = Partnerships for Health Systems Improvement, QI = quality improvement.

data point beyond the control limits, or 8 sequential points on 1 side of the mean, in either direction..$^{20,21}$

We evaluated linear trends for changes in patient characteristics, care practices and outcomes using $F$ or Wald $\chi^{2}$ tests from lin- ear or logistic regression models with the 1-year periods used as a continuous variable. We adjusted models, using a generalized estimating equation approach (to account for clustering within site) with symmetric covariance structure, for the most common 
variables known to be associated with the outcomes: gestational age in weeks (as a categorical variable), sex, multiple births, small for gestational age, outborn status, Score for Neonatal Acute Physiology version II greater than $20,{ }^{22}$ and antenatal steroids. We conducted trend analysis based on infants with nonmissing values of covariates and outcomes. We did not impute data, as more than $96 \%$ of infants had complete data. ${ }^{23}$

We also analyzed trends in the primary outcome using interrupted time series models to determine whether there were different effects in the 3 epochs. We used generalized estimated equations to examine linear trends in 3-month periods (to allow sufficient time points). ${ }^{24}$ We analyzed the change in trends for the 3 epochs.

We conducted sensitivity analyses for survival and survival without major morbidity for the 22 hospitals that participated throughout the 14-year period to evaluate the potential effect of changes in participating hospitals over time.
We carried out data management and statistical analyses using SAS version 9.3 and $\mathrm{R}$ version 3.2.2. We regarded a 2-sided $p$ value of less than 0.05 as statistically significant.

\section{Ethics approval}

We obtained waivers of individual informed consent from institutional ethics or quality-improvement committees from all participating hospitals.

\section{Results}

Of the 54923 infants born between 23 and 32 weeks' gestation and admitted to participating NICUs during the study period, 4092 infants $(7.5 \%)$ were excluded; $532(1.0 \%)$ were moribund on admission, 1936 (3.5\%) had major congenital anomalies, 62 (0.1\%) had missing gestational age or birth weight, and 1562 (2.8\%) were admitted more than 7 days after birth (Appendix 1, eFigure 1, available

Table 1: Maternal and infant characteristics of very preterm infants admitted to neonatal intensive care units in Canada, 2004-2017

\begin{tabular}{|c|c|c|c|c|c|}
\hline Characteristic & $\begin{array}{l}\text { Epoch } 1(2004-2008) \\
\qquad n=15060^{\star}\end{array}$ & $\begin{array}{c}\text { Epoch } 2(2009-2012) \\
\quad n=15692^{\star}\end{array}$ & $\begin{array}{l}\text { Epoch } 3(2013-2017) \\
\qquad n=20079^{\star}\end{array}$ & $\begin{array}{c}p \text { value } \\
\text { for epochst }\end{array}$ & $\begin{array}{l}p \text { value } \\
\text { for linear } \\
\text { trend } \neq\end{array}$ \\
\hline \multicolumn{6}{|l|}{ Maternal } \\
\hline Age, $y r$, mean \pm SD & $30.2 \pm 6.0$ & $30.7 \pm 5.9$ & $31.3 \pm 5.8$ & $<0.001$ & $<0.001$ \\
\hline Hypertension & $\begin{array}{l}n=14750 \\
2676(18.1)\end{array}$ & $\begin{array}{c}n=15200 \\
2912(19.2)\end{array}$ & $\begin{array}{c}n=19524 \\
3681(18.9)\end{array}$ & 0.07 & 0.1 \\
\hline Prenatal care & $\begin{array}{c}n=14440 \\
14080(97.5)\end{array}$ & $\begin{array}{c}n=14577 \\
14314(98.2)\end{array}$ & $\begin{array}{c}n=19307 \\
18702(96.9)\end{array}$ & $<0.001$ & $<0.001$ \\
\hline Chorioamnionitis & $\begin{array}{c}n=13678 \\
1764(12.9)\end{array}$ & $\begin{array}{c}n=12183 \\
2115(17.4)\end{array}$ & $\begin{array}{c}n=14758 \\
3081(20.9)\end{array}$ & $<0.001$ & $<0.001$ \\
\hline \multicolumn{6}{|l|}{ Infant } \\
\hline Gestational age, wk, median (IQR) & $29(27-31)$ & $30(27-31)$ & $30(27-31)$ & 0.9 & $>0.9$ \\
\hline Gestational age group, wk & & & & $<0.001$ & \\
\hline $23-25$ & $1729(11.5)$ & $1906(12.1)$ & $2630(13.1)$ & & \\
\hline $26-28$ & $4101(27.2)$ & $4014(25.6)$ & $5042(25.1)$ & & \\
\hline $29-32$ & $9230(61.3)$ & $9772(62.3)$ & $12407(61.8)$ & & \\
\hline Birth weight, g, mean \pm SD & $1324 \pm 451$ & $1328 \pm 454$ & $1325 \pm 454$ & 0.7 & 0.8 \\
\hline Sex, male & $\begin{array}{c}n=15036 \\
8031(53.4)\end{array}$ & $\begin{array}{c}n=15671 \\
8645(55.2)\end{array}$ & $\begin{array}{c}n=20054 \\
10989(54.8)\end{array}$ & 0.005 & 0.02 \\
\hline Singleton & $\begin{array}{c}n=15008 \\
10338(68.9)\end{array}$ & $\begin{array}{c}n=15683 \\
10679(68.1)\end{array}$ & $14038(69.9)$ & 0.001 & 0.02 \\
\hline Small for gestational age & $\begin{array}{l}n=15037 \\
1429(9.5)\end{array}$ & $\begin{array}{l}n=15680 \\
1530(9.8)\end{array}$ & $\begin{array}{l}n=20059 \\
1971(9.8)\end{array}$ & 0.6 & 0.3 \\
\hline Minor congenital malformation & $2423(16.1)$ & $2472(15.8)$ & $3249(16.2)$ & 0.5 & 0.8 \\
\hline SNAP-II score > 20 & $\begin{array}{l}n=15051 \\
2690(17.9)\end{array}$ & $\begin{array}{l}n=15546 \\
2527(16.3)\end{array}$ & $\begin{array}{l}n=19900 \\
2954(14.8)\end{array}$ & $<0.001$ & $<0.001$ \\
\hline
\end{tabular}


at www.cmaj.ca/lookup/suppl/doi:10.1503/cmaj.190940/-/DC1). The proportions of excluded patients remained similar across study years. The final study population included 50831 infants. Maternal and infant characteristics are presented in Table 1. During the study period, maternal age and the rate of chorioamnionitis increased. There was a small but significant increase in the proportion of male and singleton infants and a decrease in infants with Score for Neonatal Acute Physiology version II greater than 20 (Table 1). Infant outcomes are presented in Table 2.

\section{Survival without major morbidity}

Survival without major morbidity increased with increasing gestational age and for all gestational age groups over the entire study period (Figure 2). Between 2004 (first 6 months) and 2017 (last 6 months), survival without major morbidity increased from $7.3 \%(10 / 137)$ to $12.9 \%(38 / 294)$ for infants born at $23-25$ weeks' gestation and from $32.0 \%(113 / 353)$ to $60.0 \%$ (282/470) for infants born at 26-28 weeks' gestation. Trend analysis confirmed this improvement for every gestational age group and in every epoch (Appendix 1, eTable 1).

For all infants, there was special-cause variation (increase) in survival without major morbidity from 2004 (first 6 months) to 2017 (last 6 months) (Appendix 1, eFigure 2; 56.6\% [669/1183] to $70.9 \%$ [1424/2009]). The annual trend in survival without major morbidity increased significantly (adjusted odds ratio [OR] 1.08, 95\% confidence interval $[\mathrm{Cl}] 1.06-1.10)$ and the odds of survival without major morbidity increased with each epoch (Appendix 1, eTable 2). Interrupted time series analyses showed that all 3 epochs were associated with improving outcomes (all slopes $>0, p<0.002$ ). There was no significant difference in the slope of change across the 3 epochs (Appendix 1, eFigure 3 ).

\section{Survival by gestational age group}

Survival also increased with increasing gestational age. There was special-cause variation (increase) in survival among infants born at 23-25 weeks' gestational age (Figure 3), confirmed in trend analysis (adjusted OR for yearly change in survival among infants born at 23-25 weeks' gestation, 1.03, 95\% Cl 1.02-1.05) (Appendix 1, eTable 1), but not among infants born at more than 25 weeks' gestation.

\section{Neonatal morbidities}

Trend analysis showed special-cause variations (decreases) in bronchopulmonary dysplasia, retinopathy of prematurity and late-onset sepsis (Figure 4) (Appendix 1, eTable 1). There was special-cause variation (decrease) and a significant decreasing trend in the rate of the composite outcome of death or bronchopulmonary dysplasia (adjusted OR for yearly change, $0.94,95 \% \mathrm{Cl}$ 0.92-0.97) (Appendix 1, eFigure 4).

There were no special-cause variations in severe neurologic injury, necrotizing enterocolitis and early-onset sepsis (Figure 4). However, trend analysis showed a significant reduction in the rate of necrotizing enterocolitis (Appendix 1, eTable 2). Yearly trend analysis stratified by gestational age strata indicated that necrotizing enterocolitis was significantly reduced among infants born at 26 to 32 weeks' gestation, and severe neurologic injury was significantly reduced only among infants born at 26 to 28 weeks' gestation (Appendix 1, eTable 1).

\section{Care practices}

There were special-cause variations (increase) in the proportions of infants delivered at perinatal centres who were normothermic on admission (44.8\%[520/1160] to $67.5 \%[1316 / 1951])$ and

Table 2: Outcomes of very preterm infants admitted to neonatal intensive care units in Canada, 2004-2017

\begin{tabular}{|c|c|c|c|c|c|}
\hline \multirow[b]{2}{*}{ Outcome } & \multicolumn{3}{|c|}{ No. $(\%)$ of infants } & \multirow[b]{2}{*}{$\begin{array}{c}p \text { value } \\
\text { for epochs } \dagger\end{array}$} & \multirow{2}{*}{$\begin{array}{l}p \text { value } \\
\text { for linear } \\
\text { trend } \ddagger\end{array}$} \\
\hline & $\begin{array}{l}\text { Epoch } 1 \text { (2004-2008) } \\
\qquad n=15060^{\star}\end{array}$ & $\begin{array}{l}\text { Epoch } 2 \text { (2009-2012) } \\
\qquad n=15692^{\star}\end{array}$ & $\begin{array}{c}\text { Epoch } 3 \text { (2013-2017) } \\
n=20079^{\star}\end{array}$ & & \\
\hline $\begin{array}{l}\text { Survival without major } \\
\text { morbidity }\end{array}$ & $9035(60.0)$ & $10063(64.1)$ & $13886(69.2)$ & $<0.001$ & $<0.001$ \\
\hline Bronchopulmonary dysplasia & $\begin{array}{c}n=14129 \\
2937(20.8)\end{array}$ & $\begin{array}{l}n=14721 \\
2665(18.1)\end{array}$ & $\begin{array}{l}n=18954 \\
2862(15.1)\end{array}$ & $<0.001$ & $<0.001$ \\
\hline Severe neurologic injury & $\begin{array}{c}n=11593 \\
1531(13.2)\end{array}$ & $\begin{array}{l}n=12775 \\
1559(12.2)\end{array}$ & $\begin{array}{c}n=16531 \\
1804(10.9)\end{array}$ & $<0.001$ & $<0.001$ \\
\hline Necrotizing enterocolitis & $\begin{array}{c}n=14920 \\
716(4.8)\end{array}$ & $\begin{array}{l}n=15634 \\
728(4.7)\end{array}$ & $\begin{array}{l}n=20067 \\
805(4.0)\end{array}$ & 0.001 & $<0.001$ \\
\hline $\begin{array}{l}\text { Severe retinopathy of } \\
\text { prematurity }\end{array}$ & $\begin{array}{l}n=6605 \\
760(11.5)\end{array}$ & $\begin{array}{l}n=6464 \\
641(9.9)\end{array}$ & $\begin{array}{l}n=8533 \\
793(9.3)\end{array}$ & $<0.001$ & $<0.001$ \\
\hline Early-onset sepsis & $202(1.3)$ & $218(1.4)$ & $342(1.7)$ & 0.007 & $<0.001$ \\
\hline Late-onset sepsis & $2616(17.4)$ & $2248(14.3)$ & $2212(11.0)$ & $<0.001$ & $<0.001$ \\
\hline Survival & $14027(93.1)$ & $14629(93.2)$ & $18866(94.0)$ & 0.002 & 0.001 \\
\hline
\end{tabular}




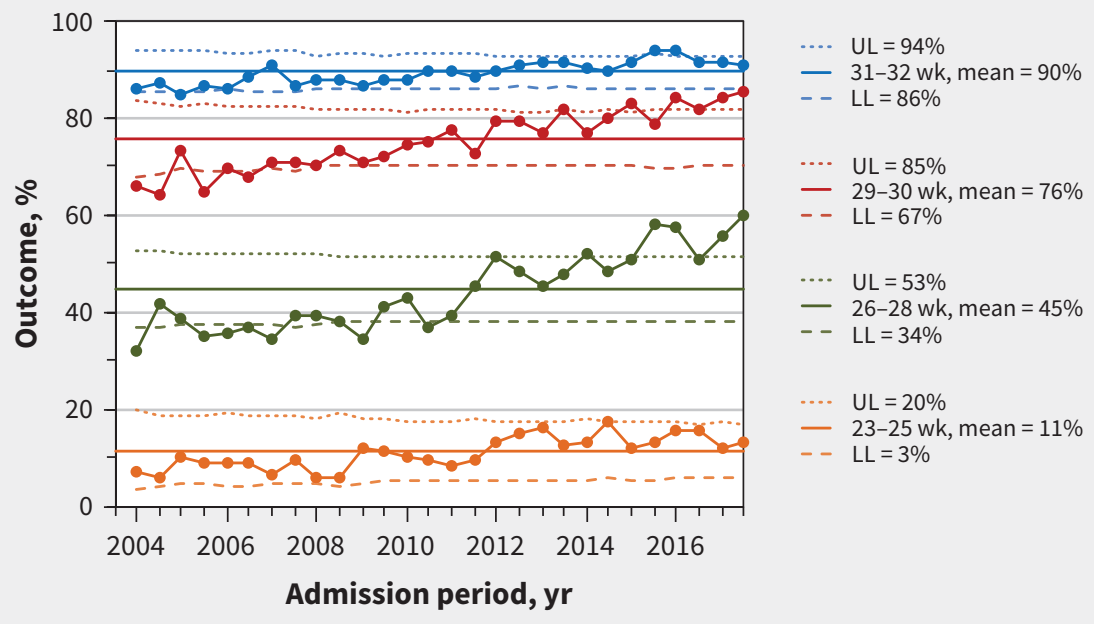

Figure 2: Survival to discharge without major morbidity. Process-control charts for survival to discharge without major morbidity by gestational age group. Means (solid lines) and upper (UL) and lower (LL) control limits (dotted and dashed lines) are calculated using 2004-2017 data. Margins of the limits were recalculated for each period based on the number of infants per 6-month period, and the reported UL and LL correspond to the first 6 months of 2004. Large dots show the percentage of infants admitted each 6-month period who survived without major morbidity. The percentages are among all infants within the gestational age group. The total numbers of infants in each group are 6265 for 23-25 weeks, 13157 for 26-28 weeks, 12868 for 29-30 weeks and 18541 for 31-32 weeks. The median (range) numbers of infants for each 6-month period are 229 (137-294) for 23-25 weeks, 485 (353-532) for 26-28 weeks, 490 (253-550) for 29-30 weeks and 700 (440-820) for 31-32 weeks.

exposed to antenatal steroids (83.6\% [904/1081] to $88.1 \%$ [1747/1983]) (Figure 5). There were special-cause variations (decreases) in pulmonary surfactant use $(52.8 \%[625 / 1183]$ to 42.7\% [857/2009]).

\section{Sensitivity analysis}

Sensitivity analyses using the survival and survival without major morbidity results of 22 units that participated during the entire study period were similar and are reported in Appendix 1, eTable 3. Similar to findings in the main analysis, the annual trend in survival without major morbidity among infants born at 23-32 weeks' gestation increased significantly (adjusted OR 1.08, 95\% Cl 1.05-1.10) and the odds of survival without major morbidity increased with each epoch. The annual trend in survival increased significantly among infants born at 23-25 weeks' gestation (adjusted OR $1.03,95 \% \mathrm{Cl}$ 1.01-1.05), but was not significant among infants born at more than 25 weeks.

\section{Interpretation}

Participation in the EPIQ program was associated with a $25 \%$ increase in survival without major morbidity among very preterm infants and a $5 \%$ increase in survival among infants born at 23-25 weeks' gestation. Participation in the program was also associated with reductions in specific morbidities, including bronchopulmonary dysplasia, retinopathy of prematurity, late-onset sepsis and necrotizing enterocolitis. These improvements occurred concomitantly with improvements in care practices including use of antenatal steroids, management of hypothermia, and more targeted use of pulmonary surfactant. Our results show that a self-learning, long-term strategy of deliberate and measured process changes can yield sustained benefits.

Quality improvement is a continually evolving process. Each epoch in our study had a different focus, informed from previous results. Epoch 1 (2003-2005) was a clusterrandomized trial that targeted single outcomes and showed significant reductions in bronchopulmonary dysplasia (15\%) and late-onset sepsis (44\%) compared with the control group. ${ }^{11}$ From 2005 to 2007, we disseminated practice guidelines from Epoch 1 to other NICUs. We found continued improvement among some NICUs that participated in the Epoch 1 study, but not others. This was consistent with reports that practice guidelines without active engagement have limited impact. 25,26

Epoch 2 (2008-2012) was a pre-post cohort study targeting multiple outcomes (mortality and the 5 major morbidities) simultaneously and emphasizing context and facilitation to change individual behaviour and organizational culture. ${ }^{12}$ We showed a significant increase in survival without major morbidity (adjusted OR 1.59, 95\% $\mathrm{Cl} 1.26-1.96$ ) and reductions in retinopathy (adjusted OR $0.60,95 \% \mathrm{Cl} 0.45-0.79$ ), necrotizing enterocolitis (adjusted OR $0.73,95 \% \mathrm{Cl} 0.52-0.98$ ) and 

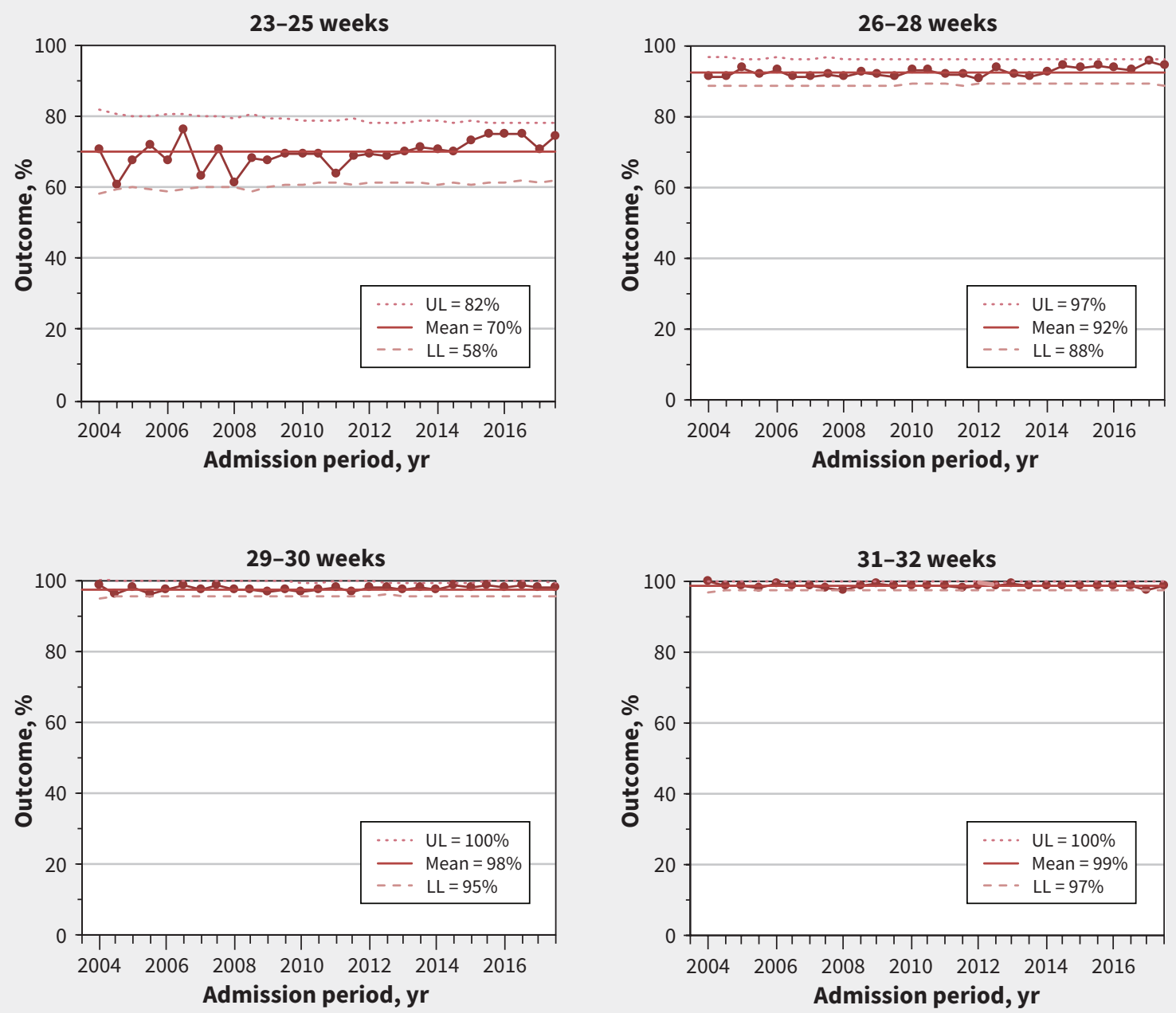

Figure 3: Survival to discharge. Process-control charts for overall survival to discharge by gestational age group. Means (solid lines) and upper (UL) and lower (LL) control limits (dotted and dashed lines) are calculated using 2004-2017 data. Margins of the limits were recalculated for each period based on the number of infants per 6-month period, and the reported UL and LL correspond to the first 6 months of 2004. Large dots show the percentage of infants admitted each 6-month period who survived to discharge. The percentages are among all infants within the gestational age group. The total numbers of infants in each group are 6265 for 23-25 weeks, 13157 for 26-28 weeks, 12868 for 29-30 weeks and 18541 for 31-32 weeks. The median (range) numbers of infants for each 6-month period are 229 (137-294) for 23-25 weeks, 485 (353-532) for 26-28 weeks, 490 (253-550) for 29-30 weeks and 700 (440-820) for 31-32 weeks.

late-onset sepsis (adjusted OR $0.63,95 \% \mathrm{Cl} 0.48$ to 0.82 ). ${ }^{12}$ However, we also observed that infants benefited more if they were born at 25 weeks' gestation or less, and hypothesized that targeting specific patient groups and processes of care may yield benefits.

Epoch 3 (2013-2017) targeted specific patient groups and processes of care, such as the first week after birth, avoiding mechanical ventilation, enhancing feeding and nutrition, supporting developmental care, and conducting routine audits of sentinel events; and encouraged inter-NICU visits to facilitate mutual learning. ${ }^{14}$ We observed further improvements in survival without major morbidity (adjusted OR 1.38, 95\% Cl 1.19-1.63).
Our results from interrupted time series analyses showed that all 3 epochs were associated with similar and continuous improvements in outcomes. Consistent with our findings, a retrospective cohort study involving preterm neonates born at less than 29 weeks' gestation among 10 high-income countries participating in the International Network for Evaluation of Outcomes between 2007 and 2015 reported that neonatal outcomes improved most in Canada, and Canada moved rank from last to second best. ${ }^{27}$ Canada's quality-improvement program was proposed as one reason for the improvement. Today, the EPIQ program is standard practice in Canadian NICUs, and the concept has been embraced in other countries, including Australia, New Zealand, Sweden, Japan, China, Malaysia and Latin America. ${ }^{28}$ 

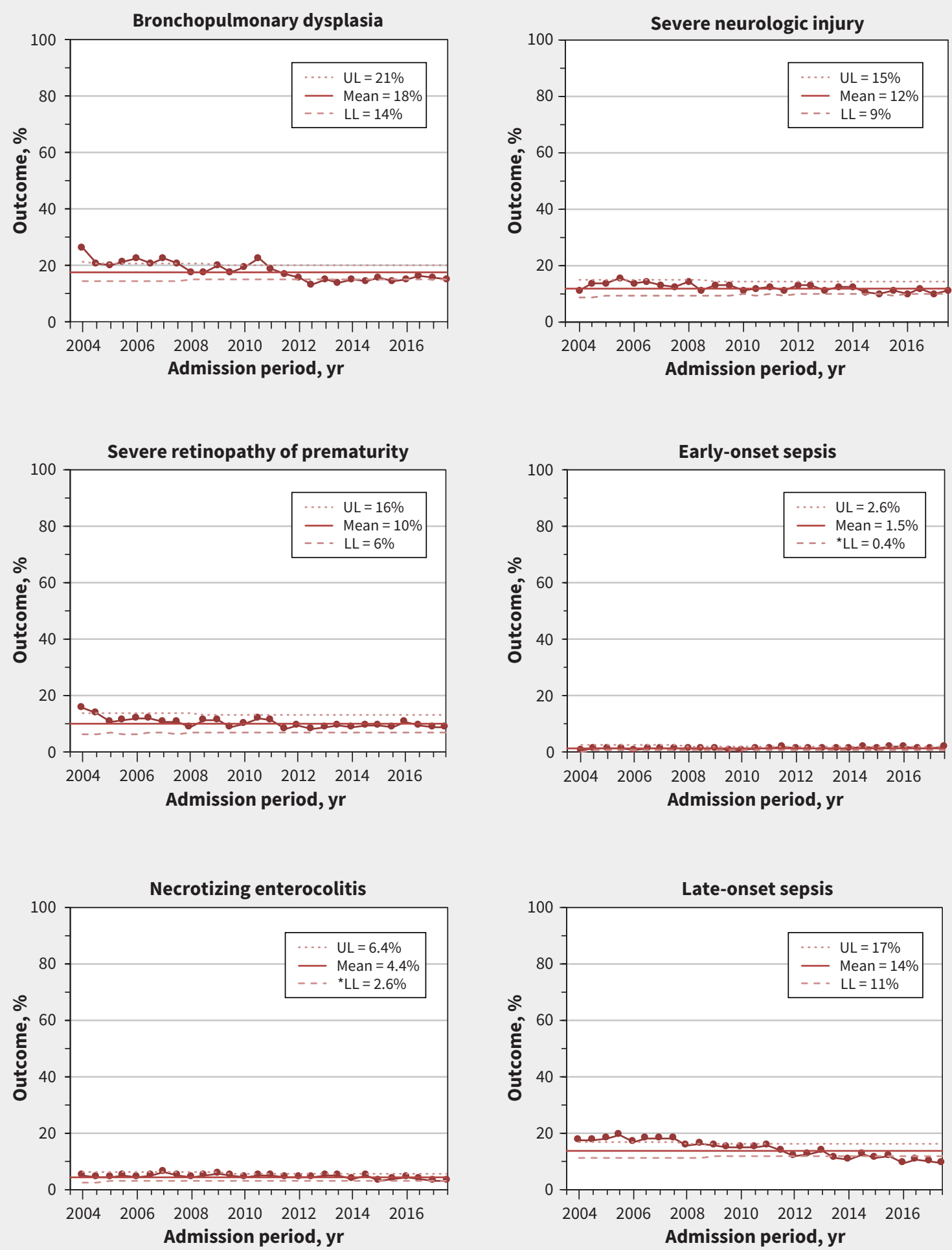

Figure 4: Process-control charts for neonatal morbidities among infants born at gestational age 23 through 32 weeks. Means (solid lines) and upper (UL) and lower (LL) control limits (dotted and dashed lines) calculated using 2004-2017 data. Margins of the limits were recalculated for each period based on the number of infants per 6-month period, and the reported UL and LL correspond to the first 6 months of 2004. Large dots show the percentage of infants admitted each 6-month period who developed the outcome. Percentage of bronchopulmonary dysplasia is among infants either alive at 36 weeks corrected, or at discharge or transfer if discharged before 36 weeks corrected $(n=47804)$. Percentage of severe neurologic injury is among infants with at least 1 documented head imaging examination (ultrasound, computed tomography or magnetic resonance imaging) $(n=40899)$. Percentage of necrotizing enterocolitis is among all infants with available data $(n=50621)$. Percentage of severe retinopathy of prematurity is among infants who had a least 1 documented eye examination $(n=21807)$. Percentage of early-onset sepsis is among all infants $(n=50831)$. Percentage of late-onset sepsis is among all infants $(n=50831)$. The median number of infants for each 6-month period was 1937 (range 538-2153). 

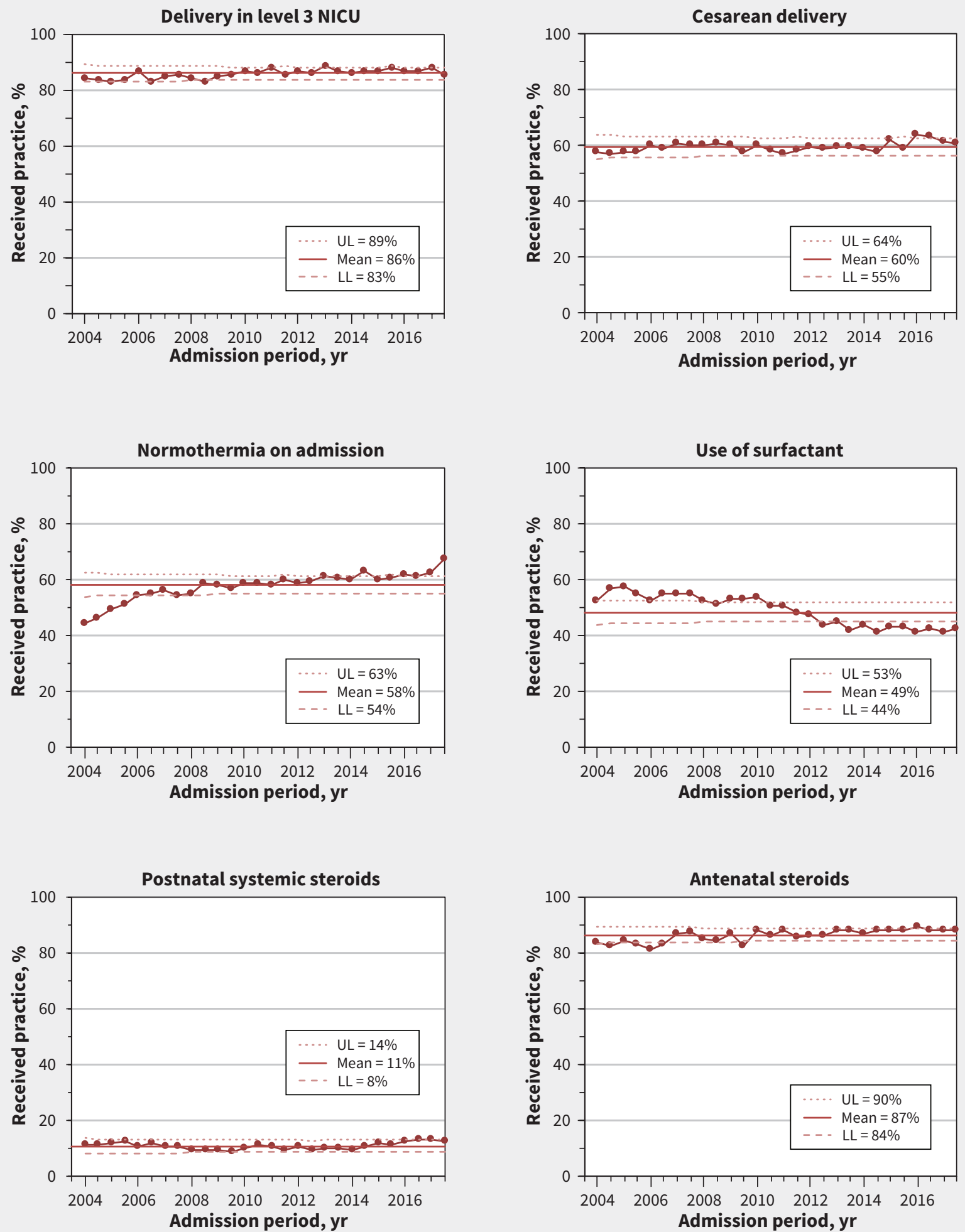

Figure 5: Process-control charts for care practices for infants born at gestational age 23 through 32 weeks. Means (solid lines) and upper (UL) and lower (LL) control limits (dotted and dashed lines) calculated using 2004-2017 data. Margins of the limits were recalculated for each period based on the number of infants per 6-month period, and the reported UL and LL correspond to the first 6 months of 2004. Large dots show the percentage of infants admitted each 6-month period who received each care practice among all eligible infants. Denominators used to calculate percentages were all infants $(n=50831)$. The median number of infants for each 6-month period was 1937 (range 1182-2152). Note: NICU = neonatal intensive care unit. 
In 2017, we expanded the EPIQ program to high-risk maternity units so that quality of care could be improved upstream and across the whole spectrum of perinatal care, from pregnancy to childbirth and infancy. Indeed, our approach should be applicable to health care beyond the NICU.

\section{Limitations}

Our study had several limitations. First, since Epochs 2 and 3 were pre-post comparisons, outcome improvements are associations that are potentially affected by residual confounding and do not confirm a causal relation between interventions and outcomes. Second, we may have overestimated survival because stillbirths, delivery room deaths and infants moribund on admission were excluded from our analyses. However, excluded neonates were not targeted by quality-improvement interventions, and their proportions have not changed over time. Third, since individual NICUs made different practice interventions at different times, we cannot provide analyses of specific interventions responsible for changes in outcomes. Fourth, controlled interrupted time series analyses could provide change compared with a reference time point; however, because of the continuous nature of our program, we used traditional measures to assess the impact of our interventions. Last, the objective of the program was to improve short-term hospital-based outcomes; long-term neurodevelopmental outcomes were not available for this cohort but are now being tracked by the Canadian Neonatal Network and should be available for future cohorts.

\section{Conclusion}

The EPIQ program in Canada has led to increases in survival and decreases in morbidity among infants born preterm, alongside the provision of improved care practices. The key lessons from the program are that a scientifically rigorous self-learning system is an effective and sustainable way to improve quality of care, and patient outcomes can be improved by doing better with what we already know and have.

\section{References}

1. Rysavy MA, Li L, Bell EF, et al. Between-hospital variation in treatment and outcomes in extremely preterm infants. N Engl J Med 2015;372:1801-11.

2. Yeo KT, Lee QY, Quek WS, et al. Trends in morbidity and mortality of extremely preterm multiple gestation newborns. Pediatrics 2015;136:263-71.

3. Johnson TJ, Patel AL, Jegier BJ, et al. Cost of morbidities in very low birth weight infants. J Pediatr 2013;162:243-49.e1.

4. Younge N, Goldstein RF, Bann CM, et al. Survival and neurodevelopmental outcomes among periviable infants. N Engl J Med 2017;376:617-28.

5. Ogrinc G, Davies L, Goodman D, et al. SQUIRE 2.0 (Standards for QUality Improvement Reporting Excellence): revised publication guidelines from a detailed consensus process. BMJ Qual Saf 2016;25:986-92.

6. Kitson A, Harvey G, McCormack B. Enabling the implementation of evidencebased practice: a conceptual framework. Qual Health Care 1998;7:149-58.

7. Canadian Neonatal Network Annual Report Committee. Canadian Neonatal Network Annual Report 2018. Toronto: Canadian Neonatal Network; 2019.
8. Canadian Neonatal Network abstractor's manual v2.1.2. Toronto: Canadian Neonatal Network; 2014. Available: www.canadianneonatalnetwork.org/ portal/CNNHome/Publications.aspx (accessed 2018 Aug. 14).

9. Shah PS, Seidlitz W, Chan P, et al. Internal audit of the Canadian Neonatal Network data collection system. Am J Perinatol 2017;34:1241-9.

10. Kramer MS, Platt RW, Wen SW, et al. A new and improved population-based Canadian reference for birth weight for gestational age. Pediatrics 2001; 108:E35.

11. Lee SK, Aziz K, Singhal N, et al. Improving the quality of care for infants: a cluster randomized controlled trial. CMAJ 2009;181:469-76.

12. Lee SK, Shah PS, Singhal N, et al. Association of a quality improvement program with neonatal outcomes in extremely preterm infants: a prospective cohort study. CMAJ 2014;186:E485-94.

13. Shah PS, Sankaran K, Aziz K, et al. Outcomes of preterm infants $<29$ weeks gestation over 10-year period in Canada: A cause for concern? J Perinatol 2012;32: 132-8.

14. Shah PS, Dunn M, Aziz K, et al. Sustained quality improvement in outcomes of preterm neonates of $<29$ weeks' gestational age: results from the Evidencebased Practice for Improving Quality Phase 3 (EPIQ-3). Can J Physiol Pharmacol 2019;97:213-21.

15. Shennan AT, Dunn MS, Ohlsson A, et al. Abnormal pulmonary outcomes in premature infants: prediction from oxygen requirement in the neonatal period. Pediatrics 1988;82:527-32.

16. Papile LA, Burstein J, Burstein R, et al. Incidence and evolution of subependymal and intraventricular hemorrhage: a study of infants with birth weights less than 1,500 gm. J Pediatr 1978;92:529-34.

17. An international classification of retinopathy of prematurity. Pediatrics 1984; 74:127-33.

18. Bell MJ, Ternberg JL, Feigin RD, et al. Neonatal necrotizing enterocolitis. Therapeutic decisions based upon clinical staging. Ann Surg 1978;187:1-7.

19. The economic aspect of patient safety in a short-term care facility [article in French]. Ottawa: Canadian Patient Safety Institute; 2012.

20. Wheeler DJ, Chambers DS. Understanding statistical process control. 2nd ed. Knoxville (TN): SPC Press; 1992.

21. Wheeler D. Understanding variation: the key to managing chaos. Knoxville (TN): SPC Press; 1993.

22. Beltempo M, Shah PS, Ye XY, et al. SNAP-II for prediction of mortality and morbidity in extremely preterm infants. J Matern Fetal Neonatal Med 2019;32:2694-701.

23. Dong Y, Peng C-YJ. Principled missing data methods for researchers. Springerplus 2013;2:222.

24. Wagner AK, Soumerai SB, Zhang F, et al. Segmented regression analysis of interrupted time series studies in medication use research. J Clin Pharm Ther 2002;27:299-309.

25. Lee SK, Aziz K, Singhal N, et al. The Evidence-based Practice for Improving Quality method has greater impact on improvement of outcomes than dissemination of practice change guidelines and quality improvement training in neonatal intensive care units. Paediatr Child Health 2015;20:e1-9.

26. Horbar JD, Rogowski J, Plsek PE, et al. Collaborative quality improvement fo neonatal intensive care. NIC/Q Project Investigators of the Vermont Oxford Network. Pediatrics 2001;107:14-22.

27. Lui K, Lee SK, Kusuda S, et al. Trends in outcomes for neonates born very preterm and very low birth weight in 11 high-income countries. J Pediatr 2019; 215:32-40.e14.

28. Cao Y, Jiang S, Zhou Q. Introducing evidence-based practice improvement in Chinese neonatal intensive care units. Transl Pediatr 2019;8:257-61. 
Competing interests: None declared. The authors further declare no competing interests for the Evidence-based Practice for Improving Quality (EPIQ) Investigators group.

This article has been peer reviewed.

Affiliations: Department of Pediatrics and Maternal-Infant Care Research Centre (Lee), Sinai Health System; Departments of Pediatrics, and Obstetrics and Gynecology, and Dalla Lana School of Public Health (Lee), University of Toronto, Toronto, Ont.; Department of Pediatrics (Beltempo), Montreal Children's Hospital and McGill University Health Centre, Montréal, Que.; Department of Pediatrics (McMillan), IWK Health Centre and Dalhousie University, Halifax, NS; Department of Pediatrics and Child Health (Seshia), Winnipeg Children's Hospital and University of Manitoba, Winnipeg, Man.; Department of Pediatrics, Foothills Medical Centre and University of Calgary (Singhal), Calgary, Alta.; Department of Paediatrics/Neonatology (Dow), Kingston Health Sciences Centre and Queen's University, Kingston, Ont.; Department of Pediatrics (Aziz), Royal Alexandra Hospital and University of Alberta, Edmonton, Alta.; Department of Pediatrics (Piedboeuf), Centre hospitalier universitaire de Québec and Université Laval, Québec, Que.; Department of Pediatrics, Sinai Health System (Shah); Department of Pediatrics, and Institute of Health Policy, Management and Evaluation (Shah), University of Toronto, Toronto, Ont.

Contributors: Shoo Lee, Marc Beltempo and Prakesh Shah conceived and designed the study, analyzed and interpreted the data, and drafted the manuscript. Douglas McMillan, Mary Seshia, Nalini Singhal, Kimberly Dow, Khalid Aziz and Bruno Piedboeuf analyzed and interpreted the data, and revised the manuscript critically for important intellectual content. All authors approved the final version of the manuscript and agreed to be accountable for all aspects of the work in ensuring that questions related to its accuracy or integrity are investigated and resolved. Shoo Lee, Marc Beltempo and Prakesh Shah had full access to all study data and take responsibility for the integrity of the data and the accuracy of the data analysis.

Funding: The EPIQ program is supported by a Canadian Institutes of Health Research (CIHR) Team Grant (CTP 87518), the Ontario Ministry of Health and Long-Term Care, and participating hospitals. Prakesh Shah holds an Applied Research Chair in Reproductive and Child Health Services and Policy Research awarded by the CIHR (APR-126340). The funding agencies had no role in the design and conduct of the study; the collection, management, analysis or interpretation of the data; the preparation, review or approval of the manuscript; or the decision to submit the manuscript for publication.

Data sharing: Data will not be available for others to use because the data transfer agreement and approvals specifically indicated that the data would not be shared with anyone.

Acknowledgements: The authors gratefully acknowledge all site investigators, abstractors and trainers of the Canadian Neonatal Network. The authors also thank the staff at the Maternal-Infant Care Research Centre at Mount Sinai Hospital, Toronto, for organizational support of the
Canadian Neonatal Network. In particular, they sincerely thank Charlene Wong, coordinator for the EPIQ program, for her tireless work in coordinating all activities related to EPIQ; and Xiang Y. Ye for conducting statistical analyses. In addition, they thank Sarah Hutchinson, from the Maternal-Infant Care Research Centre, for assistance in the preparation of this manuscript.

EPIQ Steering Committee: Shoo K. Lee (principal investigator), Prakesh S. Shah and Vibhuti Shah, Mount Sinai Hospital, Toronto, Ont.; Anne Synnes, BC Women's Hospital \& Health Centre, Vancouver, BC; Khalid Aziz, Royal Alexandra Hospital, Edmonton, Alta.; Molly Seshia, Health Sciences Centre, University of Manitoba, Winnipeg, Man.; Bruno Piedboeuf, Centre hospitalier universitaire de Québec, Sainte Foy, Que.; Kimberly Dow, Kingston Health Sciences Centre, Kingston, Ont.; Wendy Yee, Foothills Medical Centre, University of Calgary, Calgary, Alta.; Douglas McMillan, IWK Health Centre, Halifax, NS; Nalini Singhal, Foothills Medical Centre, University of Calgary, Calgary, Alta.

EPIQ Investigators: Marc Beltempo (joint first author), McGill University Health Centre, Montréal, Que.; Xiang Y. Ye, Mount Sinai Hospital, Toronto, Ont.; Julie Emberley and Akhil Deshpandey, Janeway Children's Health Centre, St. John's, NL; Jehier Afifi and Douglas McMillan, IWK Health Centre, Halifax, NS; Hala Makary, Dr. Everett Chalmers Hospital, Fredericton, NB; Roderick Canning, Moncton Hospital, Moncton, NB; Luis Monterrosa, Saint John Regional Hospital, Saint John, NB; Christine Drolet and Bruno Piedboeuf, Centre hospitalier universitaire de Québec, Sainte Foy, Que.; Keith Barrington and Anie Lapointe, CHU Sainte-Justine Hospital, Montréal, Que.; Ermelinda Pelausa, Jewish General Hospital, Montréal, Que.; Patricia Riley and Therese Perreault, Montreal Children's Hospital, Montréal, Que.; Jennifer Twiss, Amit Mukerji and Sandesh Shivananda, Hamilton Health Sciences Centre, Hamilton, Ont.; Faiza Khurshid and Kimberly Dow, Kingston Health Sciences Centre, Kingston, Ont.; David Lee, Orlando da Silva and Kevin Coughlin, London Health Sciences Centre, London, Ont.; Nicole Rouvinez-Bouali and Brigitte Lemyre, Children's Hospital of Eastern Ontario, Ottawa, Ont.; Kyong Soon Lee, Hospital for Sick Children, Toronto, Ont.; Vibhuti Shah, Mount Sinai Hospital, Toronto, Ont.; Eugene Ng and Michael Dunn, Sunnybrook Health Sciences Centre, Toronto, Ont.; Chukwuma Nwaesei, Windsor Regional Hospital, Windsor, Ont.; Ruben Alvaro, St. Boniface General Hospital, Winnipeg, Man.; Molly Seshia, Health Sciences Centre, University of Manitoba, Winnipeg, Man.; Zarin Kalapesi and Jaya Bodani, Regina General Hospital, Regina, Sask.; Sibasis Daspal, Royal University Hospital, Saskatoon, Sask.; Ayman Abou Mehrem, Khorshid Mohammad and Wendy Yee, Foothills Medical Centre, University of Calgary, Calgary, Alta.; Khalid Aziz, Royal Alexandra Hospital, Edmonton, Alta.; Sandesh Shivananda and Anne Synnes, BC Women's Hospital \& Health Centre, Vancouver, BC; Richard Taylor and Adele Harrison, Victoria General Hospital, Victoria, BC

Accepted: Nov. 29, 2019

Correspondence to: Prakesh Shah, prakeshkumar.shah@ sinaihealthsystem.ca 